\title{
Two-year effects of an interdisciplinary intervention on recovery following hip fracture in older Taiwanese with cognitive impairment
}

\author{
Yea-Ing L. Shyu ${ }^{1,2}$, Wen-Che Tsai ${ }^{3}$, Min-Chi Chen ${ }^{4}$, Jersey Liang ${ }^{5,6}$, Huey-Shinn Cheng ${ }^{7}$, Chi-Chuan $\mathrm{Wu}^{8}$, \\ Juin-Yih $\mathrm{Su}^{9}$ and Shih-Wei Chou ${ }^{10}$ \\ ${ }^{1}$ School of Nursing, Chang Gung University, Taoyuan, Taiwan \\ ${ }^{2}$ Healthy Aging Research Center, Chang Gung University, Taoyuan, Taiwan \\ ${ }^{3}$ Department of Psychiatry, National Taiwan University Hospital, Taipei, Taiwan \\ ${ }^{4}$ Department of Public Health \& Biostatistics Consulting Center, Chang Gung University, Taoyuan, Taiwan \\ ${ }^{5}$ School of Public Health, University of Michigan, Ann Arbor, MI, USA \\ ${ }^{6}$ Institute of Gerontology, University of Michigan, Ann Arbor, MI, USA \\ ${ }^{7}$ Department of Internal Medicine, Chang Gung Memorial Hospital, Taoyuan, Taiwan \\ ${ }^{8}$ Traumatological Division, Department of Orthopedics, Chang Gung Memorial Hospital, Taoyuan, Taiwan \\ ${ }^{9}$ Department of Orthopedics, Chang Gung Memorial Hospital (Keelung Branch), Keelung, Taiwan \\ ${ }^{10}$ Department of Physical Medicine \& Rehabilitation, Chang Gung Memorial Hospital, Taoyuan, Taiwan \\ Correspondence to: Professor Y-I. L. Shyu, E-mail: yeaing@mail.cgu.edu.tw
}

\begin{abstract}
Objective: This article aims to evaluate the long-term effects of an interdisciplinary intervention program on cognitively impaired older persons after hip fracture in Taiwan.

Methods: Of 160 subjects randomly assigned to control $(n=81)$ and intervention $(n=79)$ groups, 24 $(29.6 \%)$ and $27(34.2 \%)$ were cognitively impaired in the control and intervention groups, respectively, and outcomes were followed for 2 years after discharge.

Results: Among cognitively impaired subjects, more in the intervention group recovered their previous walking ability (odds ratio $[O R]=3.49$; confidence interval $[C I]=1.64$ to 7.42 ), activities of daily life performance $(\beta=18.59 ; p=0.0002)$, and more were readmitted to the hospital $(O R=4.44, C I=1.53$ to 12.89) than those in the control group during the first 2 years following discharge. Among subjects without cognitive impairment, more in the intervention group recovered their previous walking ability $(O R=2.6 ; C I=1.33$ to 5.07$)$, had fewer falls $(O R=0.47 ; C I=0.25$ to 0.86$)$, and made fewer emergency room visits $(O R=0.33$; $C I=0.11$ to 0.97$)$ during the first 2 years following discharge than those in the control group.

Conclusions: Cognitively impaired individuals benefited from our interdisciplinary intervention by improving their walking ability and physical function during the first 2 years following discharge. Specific strategies on fall prevention following hip fracture need to be further developed for cognitively impaired individuals. Copyright (C) 2011 John Wiley \& Sons, Ltd.
\end{abstract}

\begin{abstract}
Key words: cognitive impairment; hip fracture; interdisciplinary intervention; intervention effects; randomized clinical trial History: Received 28 December 2010; Accepted 9 May 2011; Published online 5 July 2011 in Wiley Online Library (wileyonlinelibrary.com).

DOI: 10.1002 /gps.2750
\end{abstract}

\section{Introduction}

Cognitive impairment, which occurs in 31-88\% of older persons with hip fracture (Holmes and House, 2000), predicts poor functional recovery and increases mortality risk after hip-fracture surgery (Heruti et al., 1999; Holmes and House,
2000; Nightingale et al., 2001; Clague et al., 2002; Gruber-Baldini et al., 2003). On the other hand, hip-fracture patients with cognitive impairment (Goldstein et al., 1997) or dementia (Huusko et al., 2000) in an active geriatric rehabilitation program achieved positive outcomes in terms of functional improvement and discharge destination, similar to those 
of cognitively normal patients. These studies examined the effects of interventions on cognitively impaired patients in developed countries. However, little is known about the differences in health trajectories of older persons with and without cognitive impairment after receiving interventions for hip fracture and even less is known about this phenomenon in Asian countries.

Taiwan differs substantially from developed countries in its healthcare system, clinical practice, case mix, culture, and social organization. For example, the majority of older people in Taiwan continue to reside with their adult married children (Lee et al., 1994). Taiwan's healthcare system differs from those in most developed countries because its national health insurance allows clients free choice of any health provider, and subacute and long-term care services are fragmented, poorly coordinated, and underdeveloped (Chuang et al., 2007). These differences require validating studies conducted in developed countries on the effects of cognitive impairment in recovery of older persons participating in intervention programs for hip fracture in Taiwan.

The health and functional outcomes of elders with hip fracture in Taiwan were found to improve after an interdisciplinary intervention program (Shyu et al., 2005). However, that clinical trial did not explore whether treatment effects differed for cognitively impaired elders. Thus, the present study was a post hoc analysis to evaluate the 2-year effects of an interdisciplinary intervention program on recovery following hip fracture for cognitively impaired older persons in Taiwan. Specifically, subjects with and without cognitive impairment participated in the intervention program or received routine care. At 1, 3, 6, 12, 18, and 24 months after hospital discharge, subjects were assessed for eight outcome variables: hip flexion ratio, recovery of walking ability, ability to perform activities of daily life (ADLs), occurrence of falls, mortality, emergency room visits, hospital readmissions, and institutionalization. In this research, we evaluated three hypotheses:

Hypothesis 1 The interdisciplinary intervention will lead to improved 2-year post-discharge health outcomes for patients without and with cognitive impairment. That is, the treatment effects will remain significant during the first 2 years when cognitive functioning is controlled.

Hypothesis 2 Cognitive functioning is significantly and positively related to outcomes.

Hypothesis 3 The effects of treatment will vary with cognitive status. That is, treatment effects will be smaller for cognitively impaired patients than for those without cognitive impairment.

\section{Methods}

Participants

Patients were included in this study if they met these criteria: (1) age 60 years or older, (2) admitted to hospital for an accidental single-side hip fracture, (3) receiving hip arthroplasty or internal fixation, (4) able to perform full range of motion (ROM) against gravity and against some or full resistance before hip fracture, (5) moderately dependent or better in ADLs before hip fracture (score $\geq 70$ on the Chinese Barthel Index $[\mathrm{CBI}]$ ), and (6) living in northern Taiwan. Inclusion criteria 4 and 5 were established to include subjects with the most potential to recover after rehabilitation. Patients were excluded if they were (1) severely cognitively impaired (score $<10$ on the Chinese Mini Mental State Examination) (Yip et al., 1992; Yip et al., 1997) or (2) terminally ill. Exclusion criterion 1 was established to exclude subjects completely unable to follow orders.

The sample was recruited from September 2001 to November 2003 and followed up during the first 2 years after discharge (Figure 1). The main reasons for patients not meeting inclusion criteria were living outside northern Taiwan, unable to perform full ROM against gravity and against some or full resistance before hip fracture, and having a CBI $<70$. Of the 162 subjects who entered the original clinical trial, 160 completed a cognitive assessment before hospital discharge and were used for this study. On the basis of the pre-discharge cognitive function assessment, subjects were categorized as cognitively impaired and assigned to the cognitive-impairment group if they had $<6$ years of education and a Chinese Mini Mental State Examination score $<21$ or had $\geq 6$ years of education and scored $<25$ (Yip et al., 1992). As shown in Figure 1, 24 subjects $(29.6 \%)$ in the control group $(n=81)$ and $27(34.2 \%)$ in the intervention group $(n=79)$ were cognitively impaired.

\section{Intervention program (intervention group)}

Current routine care of elders with hip fracture in Taiwan does not usually include continuity of care, geriatric assessment, and a well-organized interdisciplinary approach (Shyu et al., 2005). The intervention program included three components: a geriatric consultation service, a rehabilitation program, and a 


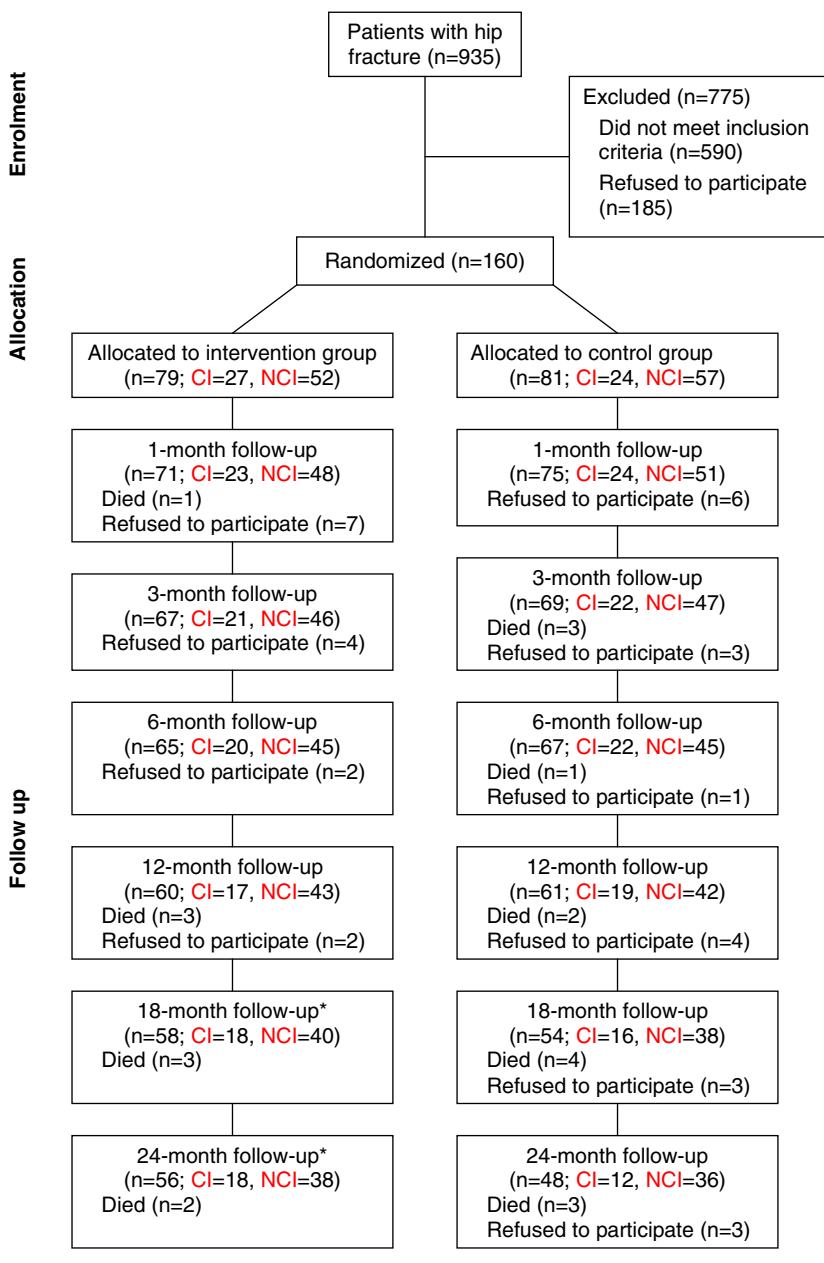

Figure 1 Study flow diagram. Sample recruitment process. *One patient refused to participate at 12 months and agreed to participate at 18 and 24 months. CI, cognitively impaired; NCI, no cognitive impairment.

discharge-planning service (Shyu et al., 2005). Geriatric consultation was provided by a geriatrician and geriatric nurses who conducted geriatric assessments/ consultations and surveyed subjects to detect potential medical and functional problems and to decrease delays before surgery. After pre-surgical and postsurgical assessments, the geriatric consultants made suggestions to the surgeon in charge about issues such as timing of surgery, infection and thromboembolic prophylaxis, postoperative nutritional management, urinary tract management, and delirium management. On the basis of these suggestions, the healthcare team (the gerontological nurse, the geriatrician, and the primary surgeon) developed or modified the care plan.

The rehabilitation component, including both inpatient and in-home rehabilitation, was delivered by a rehabilitation physician, geriatric nurses, and a physical therapist. Every intervention-group subject received both in-hospital rehabilitation (delivered during hospitalization) and in-home rehabilitation (delivered in the home setting). Rehabilitation started 1 day after surgery and continued until 3 months after discharge. Both inpatient and in-home rehabilitation included a hip fracture-oriented intervention and a general intervention program to restore deteriorated physical fitness. Average hospital stays for the intervention and control groups were 10.1 days (standard deviation $=3.7$ ) and 9.7 days (standard deviation $=$ 5.0), respectively. For the inpatient program, a geriatric nurse visited subjects four times (once a day), a physical therapist visited them two times, and a rehabilitation physician visited them once. For the inhome program, a geriatric nurse visited subjects four times during the first month and four times during the second and third months after discharge. A physical therapist visited subjects three times. Routine care (control group) included about three inpatient physical therapy sessions, with no in-home visits.

Discharge service was delivered by geriatric nurses and included a discharge assessment, necessary referrals, a home assessment, and suggested environmental modifications. Discharge assessment, which occurred during hospitalization, evaluated caregiver competence, resources, family function, patient's self-care ability, and need for community or long-term care services. On the basis of this assessment, the nurse made referrals. Before the patient's hospital discharge, a geriatric nurse visited the home to assess its environment and suggest needed changes. Follow-up clinic visits were also monitored.

Ethical considerations. This study was in compliance with the Helsinki Declaration and local legislation. Before data were collected, the study was approved for human subject research by the study hospital (Institutional Review Board, first author's hospital; approval number 89-25). Participants gave signed informed consent to participate in the study.

Outcome variables. The intervention was assessed in terms of eight outcome variables: hip flexion ratio, recovery of walking ability, ADL performance, fall occurrence, mortality, emergency room (ER) visits, hospital readmission, and institutionalization. Ratio of hip flexion and recovery of walking ability reflected physical functioning outcomes. Hip flexion ratio was defined by the ROM of the affected hip joint divided by the ROM of the unaffected hip joint. Recovery of walking ability was determined by comparing its rating before and after fracture. Walking ability was 
rated from 0 to 15 by 1 item on the CBI: 0 (immobile or $<50$ yards), 5 (wheelchair independent, including corners, $>50$ yards), 10 (walks with verbal or physical help of one person, $>50$ yards), and 15 (independent, but may use any aid, e.g., a cane, $>50$ yards). Ability to perform ADLs was measured using the CBI (Chen et al., 1995). Falls and mortality indicated clinical outcomes. Service utilization variables included ER visits, hospital readmission, and institutionalization.

\section{Procedure}

Subjects were recruited from the ER by research assistants and provided informed consent before participation. Those who agreed to participate were randomly assigned to an intervention or control group by flipping a coin. The intervention group then received routine hospital care plus the intervention program, whereas the control group received only routine hospital care. Outcomes were assessed at 1, 3, 6, 12, 18, and 24 months after hospital discharge.

Statistical analysis. Differences in baseline characteristics between the intervention and control groups were assessed by two-sample t-tests or chi-squared tests. Effects of the interdisciplinary intervention were evaluated in conjunction with cognitive functioning by a generalized estimating equation (GEE) approach. The GEE can account for possible correlations in repeated measures over time and can explore differences at different time points. Another advantage of using the GEE model is that partial information can be used; for example, data from subjects who drop out can still contribute to the estimation parameters. Therefore, this approach is especially useful in longitudinal studies where sample attrition is inevitable, but the data belonging to study participants who die or drop out within 2 years of discharge can be included in the analysis. In this study, for a given outcome variable, the GEE model included the following predictors: (i) treatment ( $1=$ intervention group, $0=$ control group); (ii) cognitive functioning ( $1=$ without cognitive impairment, $0=$ cognitively impaired), and five dummy variables representing measurements made at $3,6,12$, 18, and 24 months after hospitalization (with 1 month after discharge as the reference). Finally, variation in the treatment effect with cognitive functioning was investigated by introducing an interaction term involving these two variables (i.e., treatment $\times$ cognitive functioning) into the GEE model. GEE analyses were conducted using SAS Win 8.0 (SAS Institute, Inc., Cary, NC).

\section{Results}

The baseline characteristics of subjects in the intervention and control groups did not differ significantly, nor did those of subjects with and without cognitive impairment (Table 1). The overall intervention effects and $p$-values for the interaction and main effects were evaluated by GEE regression analysis for six of eight outcomes (Table 2). On the basis of the same GEE model, we then specifically contrasted the effects for each group (cognitive impairment intervention, cognitive impairment control, no impairment intervention, no impairment control) (Table 3). The regression coefficients for time, treatment, cognitive impairment, and interaction between intervention and cognitive impairment are presented in Table 2. To facilitate interpretation of the intervention effects, we also present in Table 2 the odds ratios (OR) with confidence intervals (CI) for significant regression coefficients of binary outcome variables, including walking recovery, hospital readmission, and recurrence of falls. The $p$-values indicate significance of the regression coefficients $(\beta)$.

This analysis showed that, after controlling for time, cognitive impairment, and interaction between the intervention and cognitive impairment, subjects in the intervention group had significantly better trajectories in ADL performance and recovery of walking ability but greater chance of hospital readmission than subjects in the control group. After controlling for covariates, subjects without cognitive impairment had significantly better trajectories in ADL performance and recovery of walking ability than cognitively impaired subjects. In terms of interaction effects, the intervention improved ADL performance for cognitively impaired subjects more than for subjects without cognitive impairment.

Overall time effects (Table 2) were evaluated using 1-month post-discharge data as baseline. Relative to this baseline, ADL performance and recovery of walking ability were significantly higher at $3,6,12,18$, and 24 months, hip flexion ratio was significantly higher at $6,12,18$, and 24 months, and hospital readmissions were higher from 6 to 12 , and from 12 to 18 months after discharge.

We then compared the outcome trajectories among different intervention $\times$ cognitive impairment groups (cognitive impairment control, cognitive impairment intervention, non-cognitive impairment control, non-cognitive impairment intervention; Table 3) using GEE analysis. For hip flexion ratio, the intervention did not significantly affect cognitively impaired subjects. However, for subjects without cognitive impairment, the intervention group had significantly 
Table 1 Demographic characteristics of patients with and without cognitive impairment recovering from hip fracture

\begin{tabular}{|c|c|c|c|c|c|c|}
\hline \multirow[b]{3}{*}{ Characteristic } & \multicolumn{3}{|c|}{ Subjects with cognitive impairment $(n=51)$} & \multicolumn{3}{|c|}{$\begin{array}{l}\text { Subjects without cognitive impairment } \\
\qquad(n=109)\end{array}$} \\
\hline & Control group & Intervention group & & Control group & Intervention group & \\
\hline & $n=24$ & $n=27$ & $p$ & $n=57$ & $n=52$ & $p$ \\
\hline Age (years), mean $\pm S D$ & $81.67 \pm 7.55$ & $81.30 \pm 6.8$ & 0.438 & $77.84 \pm 6.98$ & $75.35 \pm 8.24$ & $0.090^{\mathrm{a}}$ \\
\hline Gender, $n(\%)$ & & & 0.088 & & & 0.230 \\
\hline Female & $16(66.7)$ & $24(88.9)$ & & $40(70.2)$ & $30(57.7)$ & \\
\hline Male & 8 (33.3) & $3(11.1)$ & & $17(29.8)$ & $22(42.3)$ & \\
\hline Marital status, $n(\%)$ & & & 0.579 & & & 0.407 \\
\hline Single & $0(0)$ & $0(0)$ & & $0(0)$ & $1(1.9)$ & \\
\hline Married & $13(54.2)$ & $12(44.4)$ & & 33 (57.9) & $25(48.1)$ & \\
\hline Widowed & $11(45.8)$ & $15(55.6)$ & & $24(42.1)$ & $25(48.1)$ & \\
\hline Divorced & $0(0)$ & $0(0)$ & & $0(0)$ & $1(1.9)$ & \\
\hline Educational background, $n(\%)$ & & & 0.791 & & & 0.432 \\
\hline Illiterate & $16(66.7)$ & $20(74.1)$ & & $22(38.6)$ & $20(38.5)$ & \\
\hline Primary school & $5(20.8)$ & $5(18.5)$ & & $24(42.1)$ & $17(32.7)$ & \\
\hline High school or above & $3(12.5)$ & $2(7.4)$ & & $11(19.3)$ & $15(28.8)$ & \\
\hline Type of surgery, $n(\%)$ & & & 0.156 & & & 0.439 \\
\hline Internal fixation & $13(54.2)$ & $20(74.1)$ & & $33(57.9)$ & $34(65.4)$ & \\
\hline Arthroplasty & $11(45.8)$ & 7 (25.9) & & $24(42.1)$ & 18 (34.6) & \\
\hline $\begin{array}{l}\text { Patients with independent walking ability }{ }^{\mathrm{b}} \text {, } \\
n(\%)\end{array}$ & $16(66.7)$ & $20(74.1)$ & 0.759 & $52(91.2)$ & $48(92.3)$ & 1.000 \\
\hline Pre-fracture $A D L s^{b}$, mean $\pm S D$ & $92.92 \pm 9.08$ & $94.44 \pm 8.59$ & 0.433 & $97.54 \pm 4.34$ & $96.83 \pm 5.15$ & $0.432^{\mathrm{a}}$ \\
\hline
\end{tabular}

$p$ determined by chi-squared tests.

ADLs, activities of daily living, SD, standard deviation.

a $p$ was determined by $t$-test.

${ }^{\mathrm{b}} \mathrm{S}$ cores determined by Chinese Barthel Index.

greater hip flexion ratio than the control group $(\beta=0.11 ; p=0.005)$. Also, cognitive impairment did not affect hip flexion ratio in either the control or intervention group.
The proportion of subjects who recovered walking ability (Figure 2) was significantly higher in the intervention group (circles) than in the control group (squares) for subjects with (filled symbols) and

Table 2 Estimated regression coefficients for overall effects: time, intervention, cognitive impairment, and interaction between intervention and cognitive impairment

\begin{tabular}{|c|c|c|c|c|c|c|c|c|}
\hline \multirow[t]{2}{*}{ Outcome } & \multicolumn{5}{|c|}{ Time after discharge (months) } & \multirow[t]{2}{*}{ Treatment } & \multirow{2}{*}{$\begin{array}{l}\text { Cognitive } \\
\text { impairment }\end{array}$} & \multirow{2}{*}{$\begin{array}{c}\text { Treatment } \times \text { cognitive } \\
\text { impairment }\end{array}$} \\
\hline & 3 & 6 & 12 & 18 & 24 & & & \\
\hline $\begin{array}{l}\text { Hip flexion } \\
\text { ratio }^{\text {a }}\end{array}$ & 0.08 & $\begin{array}{c}0.17 \\
(<0.001)\end{array}$ & $\begin{array}{l}0.09 \\
(0.02)\end{array}$ & $\begin{array}{c}0.10 \\
(0.02)\end{array}$ & $\begin{array}{l}0.14 \\
(0.0007)\end{array}$ & 0.09 & 0.05 & 0.02 \\
\hline $\begin{array}{l}\text { Performance } \\
\text { of } A D L s\end{array}$ & $\begin{array}{c}7.42 \\
(<0.001)\end{array}$ & $\begin{array}{l}11.19 \\
(<0.001)\end{array}$ & $\begin{array}{c}9.46 \\
(<0.001)\end{array}$ & $\begin{array}{c}7.53 \\
(<0.001)\end{array}$ & $\begin{array}{l}6.55 \\
(0.005)\end{array}$ & $\begin{array}{l}18.59 \\
(<0.001)\end{array}$ & $\begin{array}{l}18.79 \\
(<0.001)\end{array}$ & $\begin{array}{l}-13.36 \\
(0.018)\end{array}$ \\
\hline $\begin{array}{l}\text { Walking } \\
\text { recovery }\end{array}$ & $\begin{array}{c}0.96 \\
(<0.001) \\
O R=2.6 \\
(1.71-3.97)\end{array}$ & $\begin{array}{c}1.18 \\
(0.001) \\
O R=3.24 \\
(2.04-5.14)\end{array}$ & $\begin{array}{c}1.33 \\
(0.001) \\
O R=3.78 \\
(2.26-6.30)\end{array}$ & $\begin{array}{c}0.75 \\
(0.002) \\
O R=2.11 \\
(1.33-3.37)\end{array}$ & $\begin{array}{c}0.75 \\
(0.003) \\
O R=2.13 \\
(1.30-3.47)\end{array}$ & $\begin{array}{c}1.25 \\
(0.001) \\
O R=3.49 \\
(1.64-7.42)\end{array}$ & $\begin{array}{c}0.83 \\
(0.021) \\
O R=2.30 \\
(1.13-4.67)\end{array}$ & -0.29 \\
\hline $\begin{array}{l}\text { Emergency } \\
\text { room visits } \\
\text { Hospital } \\
\text { readmissions }\end{array}$ & & $\begin{array}{l}\text { (Similar to } \\
\text { previous) }\end{array}$ & & & & & & \\
\hline $\begin{array}{l}\text { Occurrence } \\
\text { of falls }\end{array}$ & & & & & & & & -0.52 \\
\hline
\end{tabular}

Each cell presents estimated regression coefficient followed by significant $p$-value and/or estimated odds ratio followed by its $95 \%$ confidence interval.

Baseline data for all variables were 1-month post-discharge data for control group and cognitively impaired subjects.

ADLs, activities of daily living; OR, odds ratio; CI, confidence interval; ROM, range of motion.

${ }^{\mathrm{a}}$ Hip flexion ratio was defined as the ROM of the affected hip joint divided by the ROM of the unaffected hip joint. 


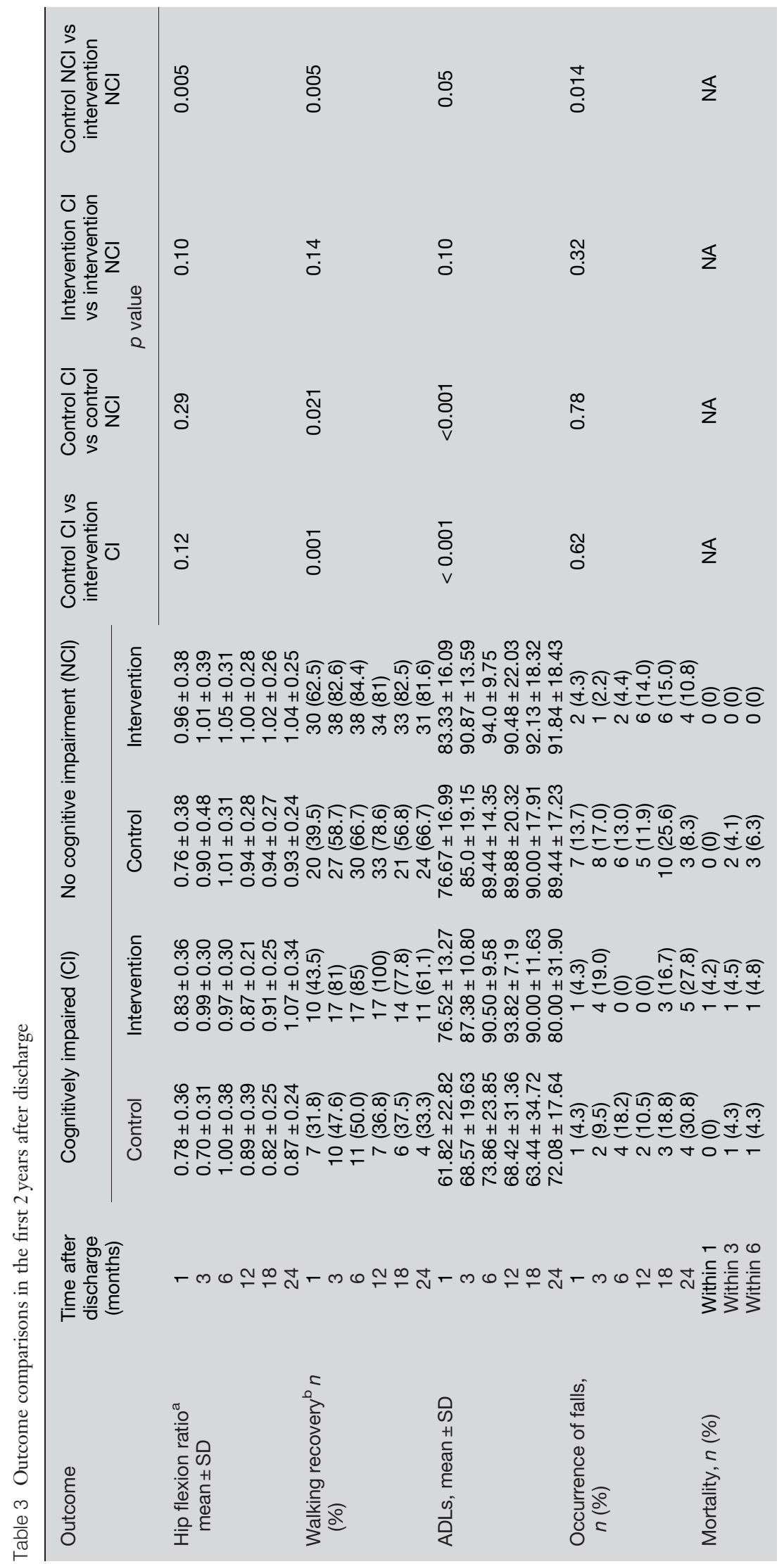




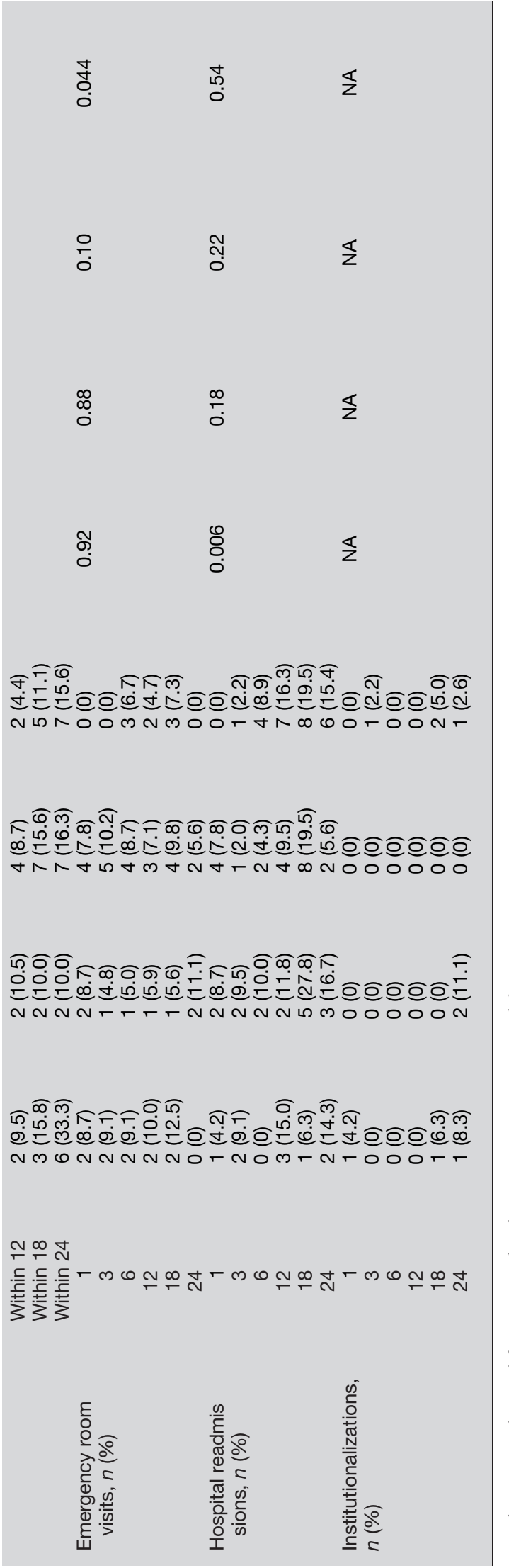

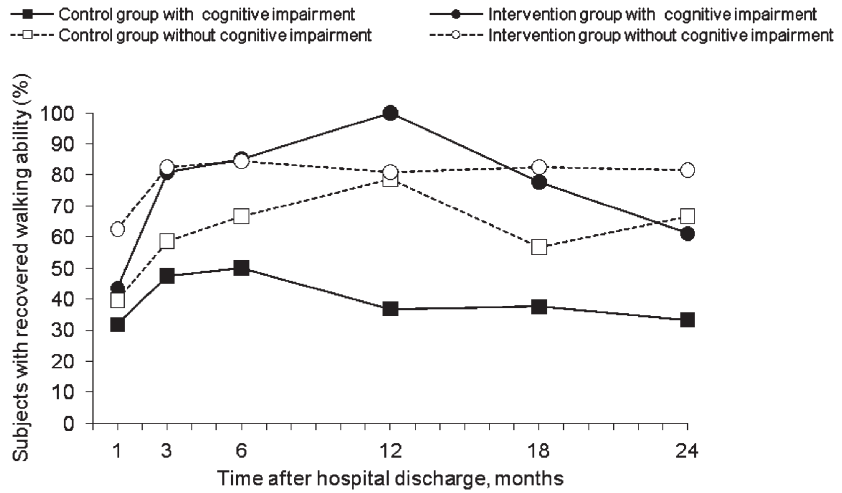

Figure 2 Changes in recovery of walking ability.

without (clear symbols) cognitive impairment. Cognitively impaired subjects in the intervention group had 3.49 greater odds of recovering their previous walking ability $(C I=1.64-7.42 ; p=0.001)$ than those in the control group. Similarly, subjects without cognitive impairment in the intervention group had 2.60 greater odds $(C I=1.33-5.07 ; p=0.005)$ of recovering walking ability than their counterparts in the control group. Furthermore, control-group subjects without cognitive impairment more easily recovered walking ability than cognitively impaired subjects $(O R=2.30 ; C I=$ $1.13-4.67 ; p=0.021)$. However, recovery of walking ability did not differ significantly between subjects with and without cognitive impairment in the intervention group.

For ADL performance, the intervention effects were different for subjects with and without cognitive impairment. Cognitively impaired subjects in the intervention group performed ADLs significantly better than their counterparts in the control group $(\beta=18.59 ; p<0.001)$. However, although subjects without cognitive impairment in the intervention group had better ADL performance at all time points than their counterparts in the control group, these differences were only close to significance $(\beta=5.24$; $p=0.05$ ). The effects of cognitive impairment were also different for subjects in the control and intervention groups. Subjects without cognitive impairment in the control group $(\beta=18.79 ; p<0.001)$ had better ADL performance than cognitively impaired subjects. However, ADL performance did not differ significantly between subjects with and without cognitive impairment in the intervention group.

For fall occurrence, subjects without cognitive impairment in the intervention group were less likely to fall $(O R=0.47 ; C I=0.25-0.86 ; p=0.014)$ during the first 2 years after discharge than those in the control 
group, but no such effect was found for cognitively impaired subjects. The intervention had no effect on fall occurrence among cognitively impaired subjects. Cognitive impairment had no effect on fall occurrence in either the control or intervention group.

For ER visits, the intervention benefited only subjects without cognitive impairment. Interventiongroup subjects without cognitive impairment were less likely $(O R=0.33 ; C I=0.11-0.97 ; p=0.044)$ to visit the ER during the first 2 years after discharge than controlgroup subjects without cognitive impairment, but no such effect was found for cognitively impaired subjects. The intervention had no effect on ER visits among cognitively impaired subjects. Cognitive impairment did not affect ER visits in either the control or intervention group. Cognitively impaired subjects in the intervention group were more likely to be readmitted to hospital $(O R=4.44, C I=1.53-12.89)$ in the first 2 years following discharge than their counterparts in the control group, but no such effect was found for subjects without cognitive impairment.

We also examined the cumulative difference in mortality among subjects. During the 2-year study period, 8 cognitively impaired subjects (six in the control, two in the intervention group) and 14 subjects without cognitive impairment (seven in the control, seven in the intervention group) died. Mortality rate did not differ significantly among the four groups (cognitive impairment control, cognitive impairment intervention, non-cognitive impairment control, non-cognitive impairment intervention). For the institutionalization rate during the first year, only one cognitively impaired subject in the control group and one subject without cognitive impairment in the intervention group were institutionalized within the first 2 months. During the second year following discharge, four cognitively impaired subjects (two in the control, two in the intervention group) and three subjects without cognitive impairment (three in the intervention group only) were institutionalized. The institutionalization rate over the 2-year period did not differ significantly among the four groups.

\section{Discussion}

This study is the first to show that a multidisciplinary intervention for hip fracture can benefit cognitively impaired non-Western older patients by improving physical functioning and ambulation, as previously observed (Goldstein et al., 1997; Heruti et al., 1999; Huusko et al., 2000). Our findings also make a unique contribution to the knowledge base by evaluating different trajectories during the first 2 years following discharge in health outcomes after an intervention for hip fracture among patients with and without cognitive impairment.

In terms of the effect of cognitive impairment, as stated in hypothesis 2 , we found that after discharge for hip-fracture surgery, cognitively impaired patients in the control group had poorer trajectories of recovery in walking ability and physical function than control-group patients without cognitive impairment. However, because our study excluded patients with severe cognitive impairment, the population from which the sample was drawn might have been less cognitively impaired than populations sampled in previous studies (Heruti et al., 1999; Matsueda and Ishii, 2000; Nightingale et al., 2001; Clague et al., 2002; Gruber-Baldini et al., 2003; Aharonoff et al., 2004; Ishida et al., 2005). Indeed, cognitive impairment has been associated with poorer physical functioning (Heruti et al., 1999; Matsueda and Ishii, 2000; Gruber-Baldini et al., 2003; Ishida et al., 2005), higher mortality (Nightingale et al., 2001; Clague et al., 2002), and more nursing home placements (Aharonoff et al., 2004). Our findings of non-significant differences in mortality and institutionalization among older patients with and without cognitive impairment might have been due to our excluding the sickest and most cognitively impaired patients who were most likely to die or be institutionalized. Thus, the numbers of mortality and institutionalization were small.

Older subjects with cognitive impairment who received our intervention after hip fracture had better trajectories for recovering walking ability and for ADL performance than their counterparts in the control group during the first 2 years following discharge, supporting hypothesis 1 . Of note, recovery of walking ability and ADL performance did not differ significantly between subjects with and without cognitive impairment in the intervention group. This finding echoes previous reports that cognitively impaired patients regained functional abilities after hip fracture and returned to the community if they received active rehabilitation (Goldstein et al., 1997; Huusko et al., 2000). Furthermore, the intervention effects on walking ability and ADL performance were even greater for cognitively impaired subjects than for those without cognitive impairment, rejecting hypothesis 3 .

We also found that cognitively impaired subjects who received the intervention were more likely to be readmitted to hospital than those in the control group. Potentially avoidable readmission rates or acute readmission within 30 days of discharge have been used as indicators for poor quality of health care (Halfon et al., 2006; Rumball-Smith and Hider, 2009). In this 
study, admission rates within 30 days following discharge did not differ significantly between cognitively impaired subjects in the control and intervention groups $(p=0.61)$. The higher overall readmission rate for cognitively impaired hip-fracture patients who received our intervention might have been due to their health problems being easier to detect and manage, because they received more home visits from nurses.

In this study, however, cognitively impaired subjects did not benefit from the intervention in preventing subsequent falls and ER visits as did subjects without cognitive impairment. Examination of hospital records showed that an important reason for ER visits of cognitively impaired subjects was subsequent falls regardless of whether they received the intervention $(n=2$ in the intervention group and $n=2$ in the control group). Despite the improved mobility of cognitively impaired patients in the intervention group, their incidence of falls was not significantly higher than that of their counterparts in the control group $(p=0.62$, Table 3$)$. We also found that ADL performance did not differ significantly between cognitively impaired subjects with and without subsequent falls during the 2 years following discharge $(t=0.26$, $p=0.80)$. Therefore, the gain in mobility for cognitively impaired patients in the intervention group did not result in more falls than in the control group and appeared to be beneficial.

Our intervention, to prevent falls, required complex skills such as assessing environmental hazards and remembering safety instructions, which were difficult for cognitively impaired patients to master. Indeed, cognitively impaired patients are viewed as the most difficult to manage in fall-prevention interventions (Detweiler et al., 2005), which are not effective in preventing falls for these patients (Shaw et al., 2003). The lack of intervention benefits on fall prevention suggests the need to develop specific strategies that consider cognitively impaired patients' level of competence, environmental factors, and rehabilitation activities to improve their recovery after hip fracture (Hedman and Grafstrom, 2001).

A limitation of our study was its single-blinded design; that is, the personnel delivering the intervention and assessing the outcomes were not blinded. However, these personnel were purposely assigned different research duties to minimize any potential bias. It is worth noting that although subjects were lost during the follow-up period, intention-to-treat analysis and on-protocol analysis produced similar results. In conclusion, despite this study's limitations and the negative impact of cognitive impairment on recovery, cognitively impaired older persons can still benefit from an interdisciplinary intervention to improve their physical function after hip fracture.

\section{Conclusions}

Cognitively impaired individuals benefited from our interdisciplinary intervention by improving their walking ability and physical function during the first 2 years following discharge. However, cognitively impaired subjects, unlike those without cognitive impairment, did not benefit from the intervention in preventing subsequent falls and ER visits. Specific fall-prevention strategies need to be further developed for cognitively impaired individuals following hip fracture.

\section{Conflict of interest}

The authors declare that they have no competing interests.

Key points

- A multidisciplinary intervention for hip fracture can benefit cognitively impaired non-Western older patients by improving physical functioning and ambulation.

- Little evidence was found on fall-prevention strategies for hip-fracture patients independent of cognitive status. Our intervention did not benefit cognitively impaired patients as it did those without impairment by preventing subsequent falls and ER visits. Specific strategies on fall prevention following hip fracture need to be further developed for individuals with cognitive impairment.

\section{Acknowledgements}

This work was funded by the National Health Research Institute, Taiwan (grant number: NHRIEX92-9023PL). The funding agency had no role in study design; in collecting, analyzing, and interpreting data; in writing the manuscript; and in deciding to submit the manuscript for publication.

\section{References}

\footnotetext{
Aharonoff GB, Barsky A, Hiebert R, Zuckerman JD, Koval KJ. 2004. Predictors of discharge to a skilled nursing facility following hip fracture surgery in New York State. Gerontology 50: 298-302. 
Chen YJ, Dai YT, Yang CT, Wang TJ, Teng YH (eds). 1995. A Review and Proposal on Patient Classification in Long-Term Care System. Department of Health, Republic of China: Taipei, Taiwan.

Chuang KY, Wu SC, Dai YT, Ma AH. 2007. Post-hospital care of stroke patients in Taipei: use of services and policy implications. Health Policy 82: 28-36.

Clague JE, Craddock E, Andrew G, Horan MA, Pendleton N. 2002. Predictors of outcome following hip fracture. Admission time predicts length of stay and in-hospital mortality. Injury 33: 1-6.

Detweiler MB, Kim KY, Taylor BY. 2005. Focused supervision of high-risk fall dementia patients: a simple method to reduce fall incidence and severity. Am J Alzheimers Dis Other Demen 20: 97-104.

Goldstein FC, Strasser DC, Woodard JL, Roberts VJ. 1997. Functional outcome of cognitively impaired hip fracture patients on a geriatric rehabilitation unit. J Am Geriatr Soc 45: 35-42.

Gruber-Baldini AL, Zimmerman S, Morrison RS, Grattan LM, Hebel JR, Dolan MM, Hawkes W, Magaziner J. 2003. Cognitive impairment in hip fracture patients: timing of detection and longitudinal follow-up. J Am Geriatr Soc 51: 1227-1236.

Halfon P, Eggli Y, Pretre-Rohrbach I, Meylan D, Marazzi A, Burnand B. 2006. Validation of the potentially avoidable hospital readmission rate as a routine indicator of the quality of hospital care. Med Care 44: 972-981.

Hedman AM, Grafstrom M. 2001. Conditions for rehabilitation of older patients with dementia and hip fracture-the perspective of their next of kin. Scand J Caring Sci 15: 151-158.

Heruti RJ, Lusky A, Barell V, Ohry A, Adunsky A. 1999. Cognitive status at admission: does it affect the rehabilitation outcome of elderly patients with hip fracture? Arch Phys Med Rehabil 80: $432-436$.

Holmes J, House A. 2000. Psychiatric illness predicts poor outcome after surgery for hip fracture: a prospective cohort study. Psychol Med 30: 921-929.
Huusko TM, Karppi P, Avikainen V, Kautiainen H, Sulkava R. 2000. Randomised, clinically controlled trial of intensive geriatric rehabilitation in patients with hip fracture: subgroup analysis of patients with dementia. BMJ 321: 1107-1111.

Ishida Y, Kawai S, Taguchi T. 2005. Factors affecting ambulatory status and survival of patients 90 years and older with hip fractures. Clin Orthop Relat Res (436): 208-215.

Lee YJ, Parish WL, Wills R. 1994. Son, daughters, and intergenerational support in Taiwan. AJS 99: 1010-1041.

Matsueda M, Ishii Y. 2000. The relationship between dementia score and ambulatory level after hip fracture in the elderly. Am J Orthop 29: 691-693.

Nightingale S, Holmes J, Mason J, House A. 2001. Psychiatric illness and mortality after hip fracture. Lancet 357: 1264-1265.

Rumball-Smith J, Hider P. 2009. The validity of readmission rate as a marker of the quality of hospital care, and a recommendation for its definition. $N Z \mathrm{Med} J$ 122(1289): 63-70.

Shaw FE, Bond J, Richardson DA, Dawson P, Steen IN, McKeith IG. 2003. Multifactorial intervention after a fall in older people with cognitive impairment and dementia presenting to the accident and emergency department: randomised controlled trial. BMJ 326: 73 .

Shyu YI, Liang J, Wu CC, Su JY, Cheng HS, Chou SW, Yang CT. 2005. A pilot investigation of the short-term effects of an interdisciplinary intervention program on elderly patients with hip fracture in Taiwan. J Am Geriatr Soc 53: 811-818.

Yip PK, Shyu YI, Liu SI, Lee JY, Chou CF, Chen RC. 1992. An epidemiological survey of dementia among elderly in an urban district of Taipei. Acta Neurologica Sinica 1: 347-354.

Yip PK, Shyu YI, Liu SI, Lee JY, Jan PL, Yang CT, Chou CF, Chen RC. 1997. The multidisplinary project of dementia study in Northern Taiwan (DSNT): background and methodology. Acta Neurological Sinica 6: 210-216. 